\title{
Regularity up to the boundary for singularly perturbed fully nonlinear elliptic equations
}

\author{
GLEYDSON C. RICARTE \\ Universidade Federal Ceará, Department of Mathematics, Fortaleza, CE-Brazil 60455-760 \\ E-mail: ricarte@mat.ufc.br \\ Jõ̃o VítoR DA SILVA \\ Universidade Federal Ceará, Department of Mathematics, Fortaleza, CE-Brazil 60455-760 \\ E-mail:vsilvamath@gmail.com
}

[Received 18 May 2014 and in revised form 19 January 2015]

In this article we are interested in studying regularity up to the boundary for one-phase singularly perturbed fully nonlinear elliptic problems, associated to high energy activation potentials, namely

$$
F\left(X, \nabla u^{\varepsilon}, D^{2} u^{\varepsilon}\right)=\zeta_{\varepsilon}\left(u^{\varepsilon}\right) \quad \text { in } \quad \Omega \subset \mathbb{R}^{n}
$$

where $\zeta_{\varepsilon}$ behaves asymptotically as the Dirac measure $\delta_{0}$ as $\varepsilon$ goes to zero. We shall establish global gradient bounds independent of the parameter $\varepsilon$.

2010 Mathematics Subject Classification: Primary 35B25, 35B65, 35D40, 35J15, 35J60, 35J75, $35 \mathrm{R} 35$.

Keywords: Fully nonlinear elliptic operators, one-phase problems, regularity up to the boundary, singularly perturbed equations, global gradient bounds.

\section{Introduction}

Throughout the last three decades or so, variational problems involving singular PDEs has received a warm attention as they often come from the theory of critical points of non-differentiable functionals. The pioneering work of Alt-Caffarelli [1] marks the beginning of such a theory by carrying out the variational analysis of the minimization problem

$$
\min \int_{\Omega}\left(|\nabla v|^{2}+\chi_{\{v>0\}}\right) d X,
$$

among competing functions with the same non-negative Dirichlet boundary condition.

Since the very beginning it has been well established that such discontinuous minimization problems could be treated by penalization methods. Indeed, Lewy-Stampacchia, KinderlehrerNirenberg, Caffarelli among others were the precursors of such an approach to the study of problem $\Delta u^{\varepsilon}=\zeta_{\varepsilon}\left(u^{\varepsilon}\right)$ over of 70 s and 80 s. Linear problems in non-divergence form was firstly considered by Berestycki et al in [2]. Teixeira in [7] started the journey of investigation into fully nonlinear elliptic equations via singular perturbation methods:

$$
F\left(X, D^{2} u^{\varepsilon}\right)=\zeta_{\varepsilon}\left(u^{\varepsilon}\right) \quad \text { in } \quad \Omega
$$


where $\zeta_{\varepsilon} \sim \varepsilon^{-1} \chi_{(0, \varepsilon)}$. The problem appears in nonlinear formulations of high energy activation models, see [6] and [7]. It can also be employed in the analysis of overdetermined problems as follows. Given $\Omega \subset \mathbb{R}^{n}$ a domain and a non-negative function $\varphi: \Omega \rightarrow \mathbb{R}$, it plays a crucial role in Geometry and Mathematical Physics the question of finding a compact hyper-surface $\partial \Omega^{\prime} \subset \Omega$ such that the following elliptic boundary value problem

$$
\left\{\begin{array}{rlll}
F\left(X, \nabla u, D^{2} u\right) & =0 & \text { in } \quad \Omega \backslash \Omega^{\prime}, \\
u & =\varphi \text { on } \partial \Omega, \\
u & =0 \text { on } \partial \Omega^{\prime},
\end{array}\right.
$$

can be solved.

Hereafter in this paper, $F: \Omega \times \mathbb{R}^{n} \times \operatorname{Sym}(n) \rightarrow \mathbb{R}$ is a fully nonlinear uniformly elliptic operator, i.e, there exist constants $\Lambda \geqslant \lambda>0$ such that

$$
\lambda\|N\| \leqslant F(X, \vec{p}, M+N)-F(X, \vec{p}, M) \leqslant \Lambda\|N\|,
$$

for all $M, N \in \operatorname{Sym}(n), N \geqslant 0, \vec{p} \in \mathbb{R}^{n}$ and $X \in \Omega$. As usual $\operatorname{Sym}(n)$ denotes the set of all $n \times n$ symmetric matrices. Moreover, we must to observe the mapping $M \mapsto F(X, \vec{p}, M)$ is monotone increasing in the natural order on $\operatorname{Sym}(n)$ and Lipschitz. Under such a structural condition, the theory of viscosity solutions provides a suitable notion for weak solutions.

DEFINITION 1.1 (Viscosity solution) For an operator $F: \Omega \times \mathbb{R}^{n} \times \operatorname{Sym}(n) \rightarrow \mathbb{R}$, we say a function $u \in C^{0}(\Omega)$ is a viscosity supersolution (resp. subsolution) to

$$
F\left(X, \nabla u, D^{2} u\right)=f(X) \text { in } \Omega,
$$

if whenever we touch the graph of $u$ by below (resp. by above) at a point $Y \in \Omega$ by a smooth function $\phi$, there holds

$$
F\left(Y, \nabla \phi(Y), D^{2} \phi(Y)\right) \leqslant f(Y) \quad(\text { resp. } \geqslant f(Y)) .
$$

Finally, we say $u$ is a viscosity solution if it is simultaneously a viscosity supersolution and subsolution.

REMARK 1.2 All functions considered in the paper will be assumed continuous in $\bar{\Omega}$, namely $C$ viscosity solutions, see Caffarellli-Cabré [3] and Teixeira [7]. However, we also can to consider $L^{p}$-viscosity notion for such a solutions, see for example Winter [8].

In [6], several analytical and geometrical properties of such a fully nonlinear singular problem (1.1) were established. Notwithstanding, regularity up to the boundary for approximating solutions has not been proven in the literature yet. This is the key goal of the present article. More precisely, we shall prove a uniform gradient estimate up to the boundary for viscosity solutions of the singular perturbation problem

$$
\left\{\begin{aligned}
F\left(X, \nabla u^{\varepsilon}, D^{2} u^{\varepsilon}\right) & =\zeta_{\varepsilon}\left(u^{\varepsilon}\right) & & \text { in } \Omega \\
u^{\varepsilon} & =\varphi & & \text { on } \partial \Omega
\end{aligned}\right.
$$

where the singular reaction term $\zeta_{\varepsilon}(s)=\frac{1}{\varepsilon} \zeta\left(\frac{s}{\varepsilon}\right)$ for some non-negative $\zeta \in C_{0}^{\infty}([0,1])$, a parameter $\varepsilon>0$, a non-negative $\varphi \in C^{1, \gamma}(\bar{\Omega})$, with $0<\gamma<1$, and, a bounded $C^{1,1}$ domain $\Omega$ (or $\partial \Omega$ for short). Throughout this paper we will assume the following bounds: $\|\varphi\|_{C^{1, \nu}(\bar{\Omega})} \leqslant Q$ and $\|\zeta\|_{L^{\infty}([0,1])} \leqslant \mathbb{B}$. 
THEOREM 1.3 (Global uniform Lipschitz estimate) Let $u^{\varepsilon}$ be a viscosity solution to the singular perturbation problem $\left(E_{\varepsilon}\right)$. Then under the assumptions $(F 1)-(F 3)$ there exists a constant $C=$ $C(n, \lambda, \Lambda, b, \propto, B, \Omega)>0$ independent of $\varepsilon$, such that

$$
\left\|\nabla u^{\varepsilon}\right\|_{L^{\infty}(\bar{\Omega})} \leqslant C
$$

Our new estimate allows us to obtain existence for corresponding free boundary problem (1.2), keeping the prescribed boundary value data, see Theorem 2.8. Finally, we should emphasize our estimate generalizes the local gradient bound proven in [7], see also [6] for a rather complete local analysis of such a free boundary problem.

Although we have chosen to carry out the global analysis for the homogeneous case, the results presented in this paper can be adapted, under some natural adjustments, for the non-homogeneous case,

$$
\left\{\begin{aligned}
F\left(X, \nabla u^{\varepsilon}, D^{2} u^{\varepsilon}\right) & =\zeta_{\varepsilon}\left(u^{\varepsilon}\right)+f_{\varepsilon}(X) & & \text { in } \quad \Omega, \\
u^{\varepsilon} & =\varphi & & \text { on } \quad \partial \Omega,
\end{aligned}\right.
$$

with $c \leqslant f_{\varepsilon} \leqslant c^{-1}$.

Our approach follows the pioneering work of Gurevich [4], where it is introduced a new strategy to investigate uniform estimate up to boundary of two-phase singular perturbation problems involving linear elliptic operators of type $\mathcal{L} u=\partial_{i}\left(a_{i j} \partial_{j} u\right)$. This method has been successfully applied by Karakhanyan in [5] for the one-phase problem in the case involving nonlinear singular/degenerate elliptic operators of $p$-Laplacian type $\Delta_{p} u^{\varepsilon}=\zeta_{\varepsilon}\left(u^{\varepsilon}\right)$.

\subsection{Notations and statements}

We shall introduce some notations which we will use throughout this paper.

- $n$ indicates the dimension of the Euclidean space.

- $H_{+}$is the half-space $\left\{X_{n}>0\right\}$.

- $T:=\left\{X=\left(X_{1}, \ldots, X_{n}\right) \in \mathbb{R}^{n}: X_{n}=0\right\}$ indicates the hyperplane.

- $\hat{X}$ is the vertical projection of $X$ on $T$.

- $\Gamma_{X}:=\left\{Y \in H_{+}:|Y-\hat{Y}| \geqslant \frac{1}{2}|Y-X|\right\}$ for $X \in T$.

- $B_{r}(X)$ is the ball with center at $X$ and radius $r$, and, $B_{r}$ the ball $B_{r}(0)$.

- $B_{r}^{+}(X):=B_{r}(X) \cap H_{+}$.

- $B_{r}^{\prime}(X)$ is the ball with center at $X$ and radius $r$ in $T$.

REMARK 1.4 Throughout this article Universal constants are the ones depending only on the dimension, ellipticity and structural properties of $F$, i. e., $n, \lambda, \Lambda$ and $b$.

Also, following classical notation, for constants $\Lambda \geqslant \lambda>0$ we denote by

$$
\mathcal{P}_{\lambda, \Lambda}^{+}(M):=\lambda \sum_{e_{i}<0} e_{i}+\Lambda \sum_{e_{i}>0} e_{i} \quad \text { and } \quad \mathcal{P}_{\lambda, \Lambda}^{-}(M):=\lambda \cdot \sum_{e_{i}>0} e_{i}+\Lambda \cdot \sum_{e_{i}<0} e_{i}
$$

the Pucci's extremal operators, where $e_{i}=e_{i}(M)$ are the eigenvalues of $M \in \operatorname{Sym}(n)$.

We shall introduce structural conditions that will be frequently used throughout of this paper:

(F1) (Ellipticity and Lipschitz regularity condition) For all $M, N \in \operatorname{Sym}(n), \vec{p}, \vec{q} \in \mathbb{R}^{n}, X \in \Omega$

$$
\mathcal{P}_{\lambda, \Lambda}^{-}(M-N)-b|\vec{p}-\vec{q}| \leqslant F(X, \vec{p}, M)-F(X, \vec{q}, N) \leqslant \mathbb{P}_{\lambda, \Lambda}^{+}(M-N)+b|\vec{p}-\vec{q}|
$$


(F2) (Normalization condition) We shall suppose that,

$$
F(X, 0,0)=0
$$

(F3) (Small oscillation condition) We must to assume

$$
\sup _{X_{0} \in \Omega} \Theta_{F}\left(X, X_{0}\right) \ll 1
$$

where

$$
\Theta_{F}\left(X, X_{0}\right):=\sup _{M \in \operatorname{Sym}(n) \backslash\{0\}} \frac{\left|F(X, 0, M)-F\left(X_{0}, 0, M\right)\right|}{\|M\|}
$$

REMARK 1.5 Assumption $(F 1)$ is equivalent to notion of uniform ellipticity Unif. Ellip. when $\vec{p}=\vec{q}$. The assumption (F2) is not restrictive, since we can always redefine the operator in order to check it. The smallest regime on oscillation of $F$, namely condition $(F 3)$, depends only on universal parameters.

EXAMPLE 1.6 An example which we must have in mind are the Isaacs' operators from stochastic game theory

$$
F(X, \vec{p}, M):=\sup _{\alpha \in \mathbb{Q}} \inf _{\beta \in \mathbb{B}}\left(\operatorname{tr}\left[A^{\alpha, \beta}(X) \cdot M\right]+\left\langle B^{\alpha, \beta}(X), \vec{p}\right\rangle\right)
$$

where $A^{\alpha, \beta}$ is a family of measurable $n \times n$ real symmetric matrices with small oscillation satisfying

$$
\lambda\|\xi\|^{2} \leqslant\left\langle A^{\alpha, \beta}(X) \xi, \xi\right\rangle \leqslant \Lambda\|\xi\|^{2}, \forall \xi \in \mathbb{R}^{n} \quad \text { and } \quad\left\|B^{\alpha, \beta}\right\|_{L^{\infty}(\Omega)} \leqslant b .
$$

\section{Optimal Lipschitz regularity}

In this section, we shall present the proof of Theorem 1.3. Thus let us assume the assumptions of problem $\left(E_{\varepsilon}\right)$.

We make a pause as to discuss some remarks which will be important throughout this work. Firstly it is important to highlight that is always possible to perform a change of variables to flatten the boundary. Indeed, if $\partial \Omega$ is a $C^{1,1}$ set, the part of $\Omega$ near $\partial \Omega$ can be covered with a finite collection of regions that can be mapped onto half-balls by diffeomorphisms (with portions of $\partial \Omega$ being mapped onto the "flat" parts of the boundaries of the half-balls). Hence, we can use a smooth mapping, reducing this way the general case to that one on $B_{1}^{+}$, and, the boundary data would be given on $B_{1} \cap\left\{X_{n}=0\right\}$.

Previously we start the proof of the global Lipschitz estimative, we need to assure the nonnegativity of solutions to $\left(E_{\varepsilon}\right)$. Such a result will be used several times throughout this article. This statement is a consequence of the Alexandroff-Bekelman-Pucci Maximum Principle, see [3] for more details.

LEMma 2.1 (Nonnegativity and bounds, [6] and [7]) Let $u^{\varepsilon}$ be a viscosity solution to $\left(E_{\varepsilon}\right)$. Then there exists a universal constant $C>0$ such that

$$
0 \leqslant u^{\varepsilon}(X) \leqslant C\|\varphi\|_{L^{\infty}(\bar{\Omega})} \quad \text { in } \quad \Omega .
$$

We will now establish a universal bound for the Lipschitz norm of $u^{\varepsilon}$ up to the boundary. The proof will be divided in two cases.

Case 1: Lipschitz regularity up to the boundary in the region $\left\{0 \leqslant u^{\varepsilon} \leqslant \varepsilon\right\}$. 
THEOREM 2.2 Let $u^{\varepsilon}$ be a viscosity solution to $\left(E_{\varepsilon}\right)$. For $X \in\left\{0 \leqslant u^{\varepsilon} \leqslant \varepsilon\right\} \cap B_{1 / 2}^{+}$there exists a universal constant $C_{1}>0$ independent of $\varepsilon$ such that

$$
\left|\nabla u^{\varepsilon}(X)\right| \leqslant C_{1} .
$$

Proof. We denote by

$$
\delta(X):=\operatorname{dist}\left(X,\left\{X_{n}=0\right\}\right)
$$

the vertical distance. If $\delta(X) \geqslant \varepsilon$, then $B_{\varepsilon}(X) \subset B_{1}^{+}$for $\varepsilon \ll 1$. Therefore, from local gradient bounds [6,7], there exists a universal constant $C_{0}>0$ independent of $\varepsilon$, such that

$$
\left|\nabla u^{\varepsilon}(X)\right| \leqslant C_{0} .
$$

On the other hand, if $\delta(X)<\varepsilon$, then it is sufficient to prove that there exists a universal constant $C_{0}>0$ independent of $\varepsilon$, such that

$$
u^{\varepsilon}(\hat{X}) \leqslant C_{0} \varepsilon .
$$

Indeed, suppose that (2.1) holds. Consider $h: \bar{B}_{1}^{+} \rightarrow \mathbb{R}$ to be the viscosity solution to the Dirichlet problem

$$
\left\{\begin{array}{rlrl}
F\left(Y, \nabla h, D^{2} h\right) & =0 & \text { in } & B_{1}^{+}, \\
h & =u^{\varepsilon} & \text { on } & \partial B_{1}^{+}
\end{array} .\right.
$$

From $C^{1, \alpha}$ regularity estimates up to the boundary (see for instance Theorem 3.1 in [8]), we know that $h \in C^{1, \alpha}\left(\bar{B}_{3 / 4}^{+}\right)$with the following estimate

$$
|\nabla h(Y)| \leqslant c\left(\|h\|_{L^{\infty}\left(B_{1}^{+}\right)}+\|\varphi\|_{C^{1, \gamma}\left(\overline{\left.B_{1}^{+}\right)}\right.}\right) \leqslant C \quad \text { in } \quad B_{3 / 4}^{+}
$$

and by Comparison Principle we have

$$
u^{\varepsilon} \leqslant h \text { in } B_{1}^{+} .
$$

Hence, it follows from assumption (2.1) that

$$
u^{\varepsilon}(Y) \leqslant h(Y) \leqslant h(\hat{X})+C|Y-\hat{X}| \leqslant C \varepsilon, \quad \text { if } Y \in B_{2 \varepsilon}^{+}(\hat{X}) .
$$

Then, again applying $C^{1, \alpha}$ regularity estimates, we obtain

$$
\left|\nabla u^{\varepsilon}(X)\right| \leqslant C_{0}(n, \lambda, \Lambda, b, \mathbb{B}) .
$$

In order to prove (2.1) suppose, by purpose of contradiction, there exists $\varepsilon>0$ such that

$$
u^{\varepsilon}(\hat{X}) \geqslant k \varepsilon \quad \text { for } \quad k \gg 1 .
$$

We shall denote

$$
r_{0}:=\operatorname{dist}\left(\hat{X},\left\{0 \leqslant u^{\varepsilon} \leqslant \varepsilon\right\}\right) .
$$

Consider $X_{0} \in\left\{0 \leqslant u^{\varepsilon} \leqslant \varepsilon\right\} \cap \partial B_{r_{0}}^{+}(\hat{X})$ a point to which the distance is achieved, i. e., $r_{0}=$ $\left|X_{0}-\hat{X}\right|$. Thereafter, let $\Gamma_{\hat{X}}$ be the cone with vertex at $\hat{X} \in\left\{X_{n}=0\right\}$. Suppose initially that $X_{0} \in \Gamma_{\hat{X}}$ then $B_{r_{0} / 2}\left(X_{0}\right) \subset B_{1}^{+}$. Now, let us define, $v^{\varepsilon}: B_{1} \rightarrow \mathbb{R}$ by

$$
v^{\varepsilon}(Y):=\frac{u^{\varepsilon}\left(X_{0}+\left(r_{0} / 2\right) Y\right)}{\varepsilon} .
$$




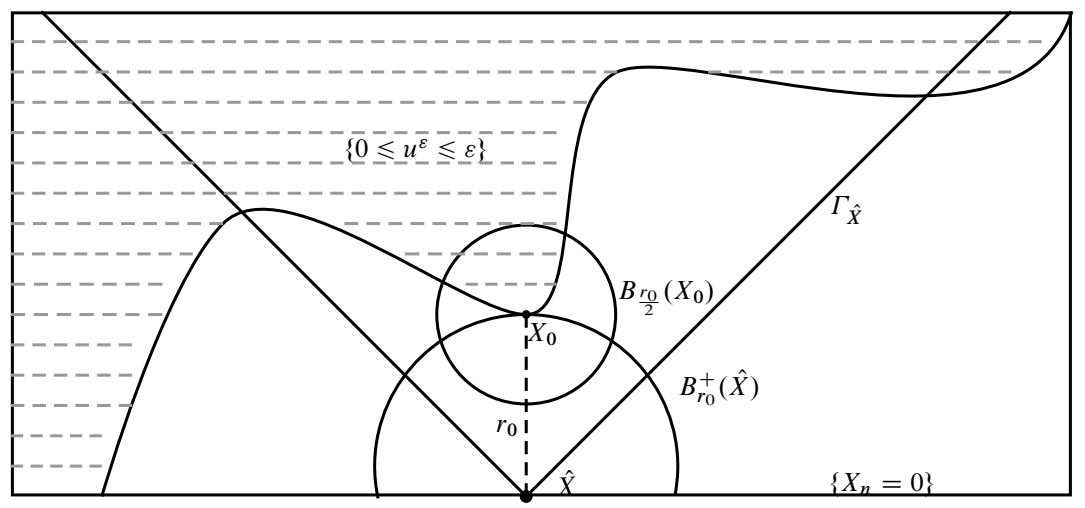

FIG. 1. Geometric argument of the case $X_{0} \in \Gamma_{\hat{X}}$

Therefore, $v^{\varepsilon}$ satisfies in the viscosity sense

$$
F_{\varepsilon}\left(X, D v^{\varepsilon}, D^{2} v^{\varepsilon}\right)=\frac{1}{\varepsilon^{2}}\left(\frac{r_{0}}{2}\right)^{2} \zeta\left(v^{\varepsilon}\right):=\mathfrak{g}(Y),
$$

where

$$
F_{\varepsilon}(X, \vec{p}, M):=\frac{1}{\varepsilon}\left(\frac{r_{0}}{2}\right)^{2} F\left(X_{0}+\frac{r_{0}}{2} Y, \frac{2 \varepsilon}{r_{0}} \cdot p, \varepsilon\left(\frac{2}{r_{0}}\right)^{2} M\right) .
$$

Now note that $\mathfrak{g} \in L^{\infty}\left(B_{1}\right)$, since $r_{0}<\varepsilon$ and $F_{\varepsilon}$ satisfies $(F 1)-(F 3)$ with constant $\tilde{b}=$ $\left(r_{0} / 2\right) b$. Moreover, since $v^{\varepsilon}(0) \leqslant 1$ it follows from Harnack inequality that

$$
v^{\varepsilon}(Y) \leqslant c \quad \text { for } \quad Y \in B_{1 / 2},
$$

i.e.,

$$
u^{\varepsilon}(X) \leqslant c \varepsilon, \quad X \in B_{r_{0} / 4}\left(X_{0}\right) .
$$

Consider now $Z \in B_{r_{0}}^{\prime}(\hat{X})$. It follows that

$$
\varphi(Z) \geqslant \varphi(\hat{X})-Q \cdot|Z-\hat{X}| \geqslant k \varepsilon-r_{0} \cdot Q \geqslant(k-Q) \varepsilon
$$

since $r_{0}<\varepsilon$. Define the scaled function $w^{\varepsilon}: B_{1}^{+} \rightarrow \mathbb{R}$,

$$
w^{\varepsilon}(Y):=\frac{u^{\varepsilon}\left(\hat{X}+r_{0} Y\right)}{\varepsilon}
$$

It readily follows that

$$
\left\{\begin{array}{rll}
F_{\varepsilon}\left(Y, \nabla w^{\varepsilon}, D^{2} w^{\varepsilon}\right)=0 & \text { in } \quad B_{1}^{+}, \\
w^{\varepsilon} \geqslant k-Q & \text { on } \quad B_{1}^{\prime} .
\end{array}\right.
$$

where $F_{\varepsilon}$ is as in (2.2). Therefore according to Lemma 2.9,

$$
w^{\varepsilon} \geqslant c(k-Q) \quad \text { in } \quad B_{3 / 4}^{+} .
$$


In other words, we have reached that

$$
u^{\varepsilon}(X) \geqslant c \varepsilon(k-Q) \quad \text { in } \quad B_{3 r_{0} / 4}^{+}(\hat{X})
$$

Hence

$$
c \varepsilon(k-Q) \leqslant u^{\varepsilon}(\xi) \leqslant c \varepsilon, \quad \forall \xi \in \partial B_{3 r_{0} / 4}^{+}(\hat{X}) \cap \partial B_{r_{0} / 4}\left(X_{0}\right),
$$

which leads to a contradiction for $k \gg 1$.

On the one hand if $X_{0} \notin \Gamma_{\hat{X}}$, choose $X_{1} \in\left\{u^{\varepsilon} \leqslant \varepsilon\right\}$ such that

$$
r_{1}:=\operatorname{dist}\left(\hat{X}_{0},\left\{u^{\varepsilon} \leqslant \varepsilon\right\}\right)=\left|\hat{X}_{0}-X_{1}\right| .
$$

From triangular inequality and the fact that $r_{1} \leqslant \frac{r_{0}}{2}$ we have

$$
\left|X_{1}-\hat{X}\right| \leqslant\left|X_{1}-\hat{X}_{0}\right|+\left|\hat{X}_{0}-\hat{X}\right| \leqslant r_{1}+r_{0} \leqslant \frac{r_{0}}{2}+r_{0}
$$

If $X_{1} \in \Gamma_{\hat{X}_{0}}$ the result follows from previous analysis. Otherwise, let $X_{2}$ be such that

$$
r_{2}:=\operatorname{dist}\left(\hat{X}_{1},\left\{u^{\varepsilon} \leqslant \varepsilon\right\}\right)=\left|\hat{X}_{1}-X_{2}\right|
$$

As before we have

$$
\left|X_{2}-\hat{X}\right| \leqslant\left|\hat{X}_{1}-X_{2}\right|+\left|\hat{X}_{1}-\hat{X}\right| \leqslant \frac{r_{0}}{4}+\frac{r_{0}}{2}+r_{0}
$$

since $r_{2} \leqslant \frac{r_{1}}{2} \leqslant \frac{r_{0}}{4}$. Observe that this process must finish up within a finite number of steps. Indeed, suppose that we have a sequence of points $X_{j} \in \partial\left\{u^{\varepsilon} \leqslant \varepsilon\right\}, \quad X_{j+1} \notin \Gamma_{\hat{X}_{j}} \quad(j=1,2, \ldots)$ satisfying

$$
r_{j+1}:=\operatorname{dist}\left(\hat{X}_{j},\left\{u^{\varepsilon} \leqslant \varepsilon\right\}\right)=\left|X_{j+1}-\hat{X}_{j}\right|,
$$

and

$$
r_{j+1} \leqslant \frac{r_{j}}{2} \leqslant \frac{r_{0}}{2^{j+1}}
$$

Thus, it follows from (2.3) that

$$
\left|X_{j}-\hat{X}\right| \leqslant r_{0}+r_{0} \sum_{i=1}^{j} \frac{1}{2^{i}} \leqslant 2 r_{0} .
$$

Therefore, up to a subsequence, $X_{j} \rightarrow \xi \in B_{2 r_{0}}^{\prime}(\hat{X})$ with $\varphi(\xi)=\varepsilon$. However,

$$
\varphi(\xi) \geqslant \varphi(\hat{X})-Q \cdot|\hat{X}-\xi| \geqslant \varepsilon(k-2 Q) \gg \varepsilon
$$

for $k \gg 1$ which drives us to a contradiction, and, hence the Assertion (2.1) is proved. 


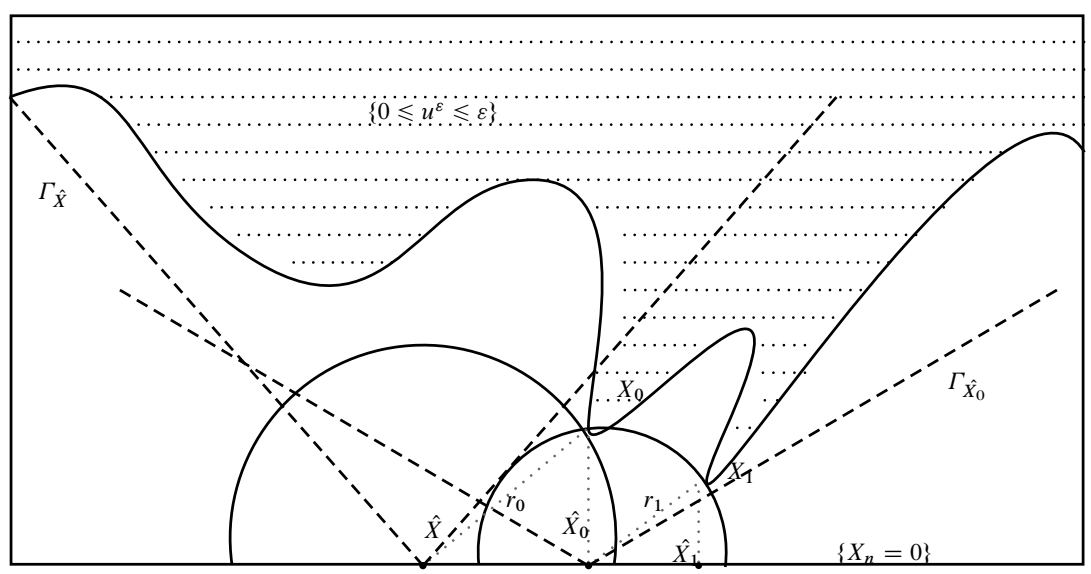

FIG. 2. Geometric argument of the inductive process

Case 2: Lipschitz regularity in the region $B_{1 / 8}^{+} \backslash\left\{u^{\varepsilon} \leqslant \varepsilon\right\}$.

THEOREM 2.3 Let $u^{\varepsilon}$ be a viscosity solution to $\left(E_{\varepsilon}\right)$. Suppose that $X \in B_{1 / 8}^{+}$satisfies $u^{\varepsilon}(X)>\varepsilon$, then there exists a constant $C_{0}=C_{0}(n, \lambda, \Lambda, b, Q)>0$ such that

$$
\left|\nabla u^{\varepsilon}(X)\right| \leqslant C_{0} .
$$

The proof of the theorem consists in analyzing three possible cases (Lemmas 2.5, 2.6, 2.7 below). Henceforth we shall use the following notation

$$
\delta_{\varepsilon}(X):=\operatorname{dist}\left(X,\left\{u^{\varepsilon} \leqslant \varepsilon\right\}\right) \text { and } \delta(X):=\operatorname{dist}\left(X,\left\{X_{n}=0\right\}\right) .
$$

The next result is decisive in our approach.

Lemma 2.4 Let $u^{\varepsilon}$ be a viscosity solution to $\left(E_{\varepsilon}\right)$ with $\varphi \in C^{1, \gamma}\left(\bar{B}_{1}^{+}\right)$. Then, for all $X \in B_{1 / 4}^{\prime}$ satisfying $u^{\varepsilon}(X)>\varepsilon$, there exists a constant $c_{0}=c_{0}(n, \lambda, \Lambda, b)>0$ such that

$$
\varphi(X) \leqslant \varepsilon+c_{0} \cdot \delta_{\varepsilon}(X) .
$$

Proof. Let us suppose for sake of contradiction that there exists an $\varepsilon>0$ and $X_{0} \in B_{1 / 4}^{\prime} \backslash\left\{u^{\varepsilon} \leqslant \varepsilon\right\}$ such that

$$
\varphi\left(X_{0}\right) \geqslant \varepsilon+k \cdot \delta_{\varepsilon}\left(X_{0}\right)
$$

holds for $k \gg 1$, large enough. Let $Z=Z_{\varepsilon} \in \partial\left\{u^{\varepsilon} \leqslant \varepsilon\right\}$ be a point to which the distance is achieved, i.e.

$$
\delta_{\varepsilon}:=\delta_{\varepsilon}\left(X_{0}\right)=\left|X_{0}-Z\right| .
$$

We have two cases to analyze: If $Z \in \Gamma_{X_{0}}$, then the normalized function $v^{\varepsilon}: B_{1}^{+} \rightarrow \mathbb{R}$ given by

$$
v^{\varepsilon}(Y):=\frac{u^{\varepsilon}\left(X_{0}+\delta_{\varepsilon} Y\right)-\varepsilon}{\delta_{\varepsilon}},
$$


satisfies

$$
F_{\varepsilon}\left(Y, \nabla v^{\varepsilon}, D^{2} v^{\varepsilon}\right)=0 \quad \text { in } \quad B_{1}^{+}
$$

in the viscosity sense, where

$$
F_{\varepsilon}(Y, \vec{p}, M):=\delta_{\varepsilon} F\left(X_{0}+\delta_{\varepsilon} Y, \vec{p}, \delta_{\varepsilon}^{-1} M\right)
$$

As in Theorem 2.2, $F_{\varepsilon}$ satisfies $(F 1)-(F 3)$ with constant $\tilde{b}=\delta_{\varepsilon} b$. Moreover,

$$
v^{\varepsilon}(Y) \geqslant 0 \quad \text { in } \quad B_{1}^{+} .
$$

Now, for any $X \in B_{\delta_{\varepsilon}}^{\prime}\left(X_{0}\right)$ we should have for $k \gg 1$,

$$
\begin{aligned}
\varphi(X) & \geqslant \varphi\left(X_{0}\right)-a \delta_{\varepsilon} \geqslant \varepsilon+k \delta_{\varepsilon}-a \delta_{\varepsilon} \\
& \geqslant \varepsilon+\frac{k}{2} \delta_{\varepsilon},
\end{aligned}
$$

i.e,

$$
\frac{\varphi\left(X_{0}+\delta_{\varepsilon} Y\right)-\varepsilon}{\delta_{\varepsilon}} \geqslant \frac{k}{2} \quad \text { in } B_{1}^{\prime}
$$

In other words,

$$
v^{\varepsilon}(Y) \geqslant c k \quad \forall Y \in B_{1}^{\prime} .
$$

Hence, from Lemma 2.9 we have that $v^{\varepsilon} \geqslant c k$ in $B_{3 / 4}^{+}$in a more precise manner,

$$
u^{\varepsilon}(X) \geqslant \varepsilon+C k \delta_{\varepsilon}, \quad X \in B_{3 \delta_{\varepsilon} / 4}^{+}\left(X_{0}\right) \text {. }
$$

From now on, let us consider $\tilde{B}:=B_{\frac{\delta_{\varepsilon}}{4}}(P)$, where $P=P_{\varepsilon}:=Z+\frac{X_{0}-Z}{4}$. If we define $\omega^{\varepsilon}:=$ $u^{\varepsilon}-\varepsilon$, then since $Z \in \partial \tilde{B}$, it follows that

$$
\begin{gathered}
F_{\varepsilon}\left(X, \nabla \omega^{\varepsilon}, D^{2} \omega^{\varepsilon}\right)=0 \text { in } \tilde{B}, \\
\omega^{\varepsilon}(Z)=u^{\varepsilon}(Z)-\varepsilon=0, \\
\frac{\partial \omega^{\varepsilon}}{\partial \nu}(Z) \leqslant\left|\nabla \omega^{\varepsilon}(Z)\right| \leqslant C .
\end{gathered}
$$

Therefore, from (2.5)-(2.7) we can apply Lemma 2.10, which gives $\omega^{\varepsilon}(P) \leqslant C_{0} \cdot \delta_{\varepsilon}$, i.e.,

$$
u^{\varepsilon}(P) \leqslant \varepsilon+C \delta_{\varepsilon} .
$$

At a point $P$ on $\partial B_{3 \delta_{\varepsilon} / 4}^{+}\left(X_{0}\right)$ we have (according to (2.4) and (2.8))

$$
\varepsilon+k c \delta_{\varepsilon} \leqslant u^{\varepsilon}(P) \leqslant \varepsilon+C_{0} \delta_{\varepsilon}
$$

which gives a contradiction if $k$ has been chosen large enough.

The second case, namely $Z \notin \Gamma_{X_{0}}$, it is treated similarly as in Theorem 2.2 and we omit the details here. 
LEMMA 2.5 Let $u^{\varepsilon}$ be a viscosity solution to $\left(E_{\varepsilon}\right)$ and $X \in B_{1 / 8}^{+}$satisfying $u^{\varepsilon}(X)>\varepsilon$ with $\delta_{\varepsilon}(X) \leqslant \delta(X)$. Then there is a universal constant $C_{0}>0$, such that

$$
\left|\nabla u^{\varepsilon}(X)\right| \leqslant C_{0}
$$

Proof. We may assume with no loss of generality that $\delta_{\varepsilon}(X) \leqslant \frac{1}{8}$. Otherwise, if we suppose that $\delta_{\varepsilon}(X)>\frac{1}{8}$, then the result would follow from [6,7]. From now on, we select $X_{\varepsilon} \in \partial\left\{u^{\varepsilon} \leqslant \varepsilon\right\}$ a point which achieves the distance, i.e.,

$$
\delta_{\varepsilon}:=\delta_{\varepsilon}(X)=\left|X-X_{\varepsilon}\right|
$$

Since

$$
\left|X_{\varepsilon}\right| \leqslant|X|+\delta_{\varepsilon} \leqslant \frac{1}{4}
$$

we must have that $X_{\varepsilon} \in B_{1 / 4}^{+} \cap\left\{u^{\varepsilon} \leqslant \varepsilon\right\}$. This way, by applying Theorem 2.2, there exists a constant $C_{1}=C(n, \lambda, \Lambda, b, Q, B)>0$ such that

$$
\left|\nabla u^{\varepsilon}\left(X_{\varepsilon}\right)\right| \leqslant C_{1}
$$

By defining the re-normalized function $v^{\varepsilon}: B_{1} \rightarrow \mathbb{R}$ as

$$
v^{\varepsilon}(Y):=\frac{u^{\varepsilon}\left(X+\delta_{\varepsilon} Y\right)-\varepsilon}{\delta_{\varepsilon}}
$$

Then, as before $v^{\varepsilon}$ satisfies

$$
\begin{aligned}
F_{\varepsilon}\left(Y, \nabla v^{\varepsilon}, D^{2} v^{\varepsilon}\right) & =0 \quad \text { in } \quad B_{1}, \\
v^{\varepsilon}\left(Y_{\varepsilon}\right) & =0, \\
\left|\nabla v^{\varepsilon}\left(Y_{\varepsilon}\right)\right| & \leqslant C_{1}, \\
v^{\varepsilon}(Y) & \geqslant 0 \quad \text { for } \quad Y \in B_{1},
\end{aligned}
$$

where

$$
F_{\varepsilon}(Y, \vec{p}, M):=\delta_{\varepsilon} F\left(X+\delta_{\varepsilon} Y, \vec{p}, \delta_{\varepsilon}^{-1} M\right) \quad \text { and } \quad Y_{\varepsilon}:=\frac{X_{\varepsilon}-X}{\delta_{\varepsilon}} \in \partial B_{1} .
$$

From (2.9)-(2.12) we are able to apply Lemma 2.10 and conclude that there exists a universal constant $c>0$ such that

$$
v^{\varepsilon}(0) \leqslant c .
$$

Moreover, from Harnack inequality

$$
v^{\varepsilon} \leqslant C_{0} \quad \text { in } \quad B_{1 / 2} .
$$

Therefore, by $C^{1, \alpha}$ regularity estimates (see Theorem 3.1 in [3]) we must have that

$$
\left|\nabla u^{\varepsilon}(X)\right|=\left|\nabla v^{\varepsilon}(0)\right| \leqslant \frac{1}{\delta_{\varepsilon}}\left\|u^{\varepsilon}-\varepsilon\right\| \leqslant C_{0},
$$

and the Lemma is proved. 
Lemma 2.6 Given $X \in B_{1 / 8}^{+}$such that $u^{\varepsilon}(X)>\varepsilon$. If $\delta(X)<\delta_{\varepsilon}(X) \leqslant 4 \delta(X)$, then

$$
\left|\nabla u^{\varepsilon}(X)\right| \leqslant C_{0}
$$

for some constant $C_{0}=C_{0}(n, \lambda, \Lambda, b, Q, B)>0$.

Proof. Similar to Lemma 2.5, we may assume that $\delta_{\varepsilon} \leqslant \frac{1}{8}$, otherwise, as in Lemma 2.5 the gradient boundedness follows from local estimates [6,7]. Define the scaled function $v^{\varepsilon}: B_{1} \rightarrow \mathbb{R}$ by

$$
v^{\varepsilon}(Y):=\frac{u^{\varepsilon}(X+\delta Y)-\varepsilon}{\delta},
$$

where $\delta=\delta(X)$. Clearly

$$
F_{\delta}\left(Y, \nabla v^{\varepsilon}, D^{2} v^{\varepsilon}\right)=0 \quad \text { in } \quad B_{1}
$$

in the viscosity sense, and, from Harnack inequality

$$
v^{\varepsilon} \leqslant C v^{\varepsilon}(0) \sim \frac{1}{\delta} \quad \text { in } B_{1 / 2}
$$

By applying once more $C^{1, \alpha}$ regularity estimates, we obtain

$$
\left|\nabla u^{\varepsilon}(X)\right|=\left|\nabla v^{\varepsilon}(0)\right| \leqslant \frac{C}{\delta} .
$$

Therefore, the idea is to find an estimate for $u^{\varepsilon}-\varepsilon$ in terms of the vertical distance $\delta(X)$. To this end, consider $h$ the viscosity solution to the Dirichlet problem

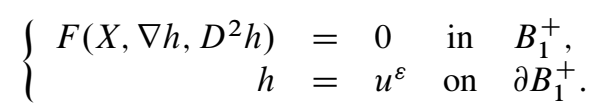

Since $0 \leqslant u^{\varepsilon} \leqslant C(n, \lambda, \Lambda, b, B)$, it follows from $C^{1, \alpha}$ estimate up to boundary that $h \in$ $C^{1, \alpha}\left(\bar{B}_{3 / 4}^{+}\right)$. Moreover

$$
|\nabla h(X)| \leqslant C\left(\|h\|_{L^{\infty}}+\|\varphi\|_{C^{1, \alpha}}\right) \leqslant C(C+Q) .
$$

From Comparison Principle, we have that

$$
u^{\varepsilon} \leqslant h \quad \text { in } \quad B_{1}^{+} .
$$

Hence,

$$
u^{\varepsilon}(X) \leqslant h(X) \leqslant h(\hat{X})+C(C+Q)|X-\hat{X}| \leqslant \varphi(\hat{X})+C(C+Q) \delta .
$$

Now, we have that $|\hat{X}| \leqslant|X|+\delta \leqslant \frac{1}{4}$, and, consequently we are able to apply Lemma 2.4 which gives

$$
\varphi(\hat{X}) \leqslant \varepsilon+c_{0} \cdot \operatorname{dist}\left(\hat{X},\left\{u^{\varepsilon} \leqslant \varepsilon\right\}\right) \leqslant \varepsilon+c_{0}\left(\delta_{\varepsilon}+\delta\right) \leqslant \varepsilon+5 c_{0} \delta .
$$

Thus, it follows from (2.15) and (2.16) that $u^{\varepsilon}(X)-\varepsilon \leqslant C_{0} \delta$, where $C_{0}:=C\left(5 c_{0}+C(C+Q)\right)$. Finally, if we apply $C^{1, \alpha}$ estimate, Harnack inequality and estimate (2.13), respectively, we end up with

$$
\left|\nabla u^{\varepsilon}(X)\right|=\left|\nabla v^{\varepsilon}(0)\right| \leqslant \frac{1}{\delta}\left\|u^{\varepsilon}-\varepsilon\right\|_{L^{\infty}\left(B_{1 / 2}\right)} \leqslant C_{0}
$$

which concludes the proof. 
LEMMA 2.7 Let $X \in B_{1 / 8}^{+}$be such that $u^{\varepsilon}(X)>\varepsilon$. If $4 \delta(X)<\delta_{\varepsilon}(X)$, then there exists a constant $C_{0}=C_{0}(n, \lambda, \Lambda, b, Q, B)>0$ such that

$$
\left|\nabla u^{\varepsilon}(X)\right| \leqslant C_{0}
$$

Proof. Initially we will consider the case when $\delta_{\varepsilon} \leqslant 1 / 8$. The following inclusion holds true: $B_{\delta_{\varepsilon} / 2}^{+}(\hat{X}) \subset B_{1 / 4}^{+} \backslash\left\{u^{\varepsilon} \leqslant \varepsilon\right\}$. In fact, if $Y \in B_{\delta_{\varepsilon} / 2}^{+}(\hat{X})$ then

$$
|Y| \leqslant|Y-X|+|X| \leqslant 2 \frac{\delta_{\varepsilon}}{2}+|X| \leqslant \frac{1}{4} .
$$

Now, using the same argument as in Lemma 2.6 (see (2.14)) we are able to estimate $u^{\varepsilon}$ in $B_{\delta_{\varepsilon} / 2}^{+}(\hat{X})$ as follows

$$
u^{\varepsilon}(Y) \leqslant u^{\varepsilon}(\hat{Y})+C(C+Q) \frac{\delta_{\varepsilon}}{2} \leqslant \varepsilon+c_{0} \cdot \operatorname{dist}\left(\hat{Y},\left\{u^{\varepsilon} \leqslant \varepsilon\right\}\right)+C(C+Q) \frac{\delta_{\varepsilon}}{2} .
$$

Since the distance function is Lipschitz continuous with Lipschitz constant 1, we have

$$
\operatorname{dist}\left(\hat{Y},\left\{u^{\varepsilon} \leqslant \varepsilon\right\}\right) \leqslant \delta_{\varepsilon}+|\hat{Y}-X| \leqslant 2 \delta_{\varepsilon} .
$$

Therefore,

$$
u^{\varepsilon}(Y) \leqslant \varepsilon+\left(2 c_{0}+\frac{C(C+Q)}{2}\right) \delta_{\varepsilon}=\varepsilon+c \delta_{\varepsilon} .
$$

By considering the function $v^{\varepsilon}(Y)=u^{\varepsilon}(Y)-\varepsilon$ in $B_{\delta_{\varepsilon} / 2}^{+}(\hat{X})$, we have that

$$
F\left(Y, \nabla v^{\varepsilon}, D^{2} v^{\varepsilon}\right)=0 \quad \text { in } \quad B_{\delta_{\varepsilon} / 2}^{+}(\hat{X})
$$

in the viscosity sense. From $C^{1, \alpha}$ estimate up to boundary and Lemma 2.1, we have

$$
\left|\nabla u^{\varepsilon}(X)\right|=\left|\nabla v^{\varepsilon}(X)\right| \leqslant C(c+Q) .
$$

On the other hand, for the case $\delta_{\varepsilon} \geqslant 1 / 8$ we have the following inclusion $B_{1 / 16}^{+}(\hat{X}) \subset B_{1} \backslash\left\{u^{\varepsilon} \leqslant \varepsilon\right\}$. In this situation, $\operatorname{since} \operatorname{supp}\left(\beta_{\varepsilon}\right)=[0, \varepsilon]$,

$$
\left\{\begin{array}{rll}
F\left(X, \nabla u^{\varepsilon}, D^{2} u^{\varepsilon}\right)=0 & \text { in } & B_{1 / 16}^{+}(\hat{X}) \\
0 \leqslant u^{\varepsilon} \leqslant C & \text { on } & \partial B_{1 / 16}^{+}(\hat{X})
\end{array}\right.
$$

and, consequently, the estimate will follow from $C^{1, \alpha}$ estimates up to the boundary.

An immediate consequence of Theorem 1.3 is the existence of solutions via compactness in the Lip-Topology for any family $\left(u^{\varepsilon}\right)_{\varepsilon>0}$ of viscosity solutions to singular perturbation problem $\left(E_{\varepsilon}\right)$. We consequently obtain

THEOREM 2.8 (Limiting free boundary problem) Let $\left(u^{\varepsilon}\right)_{\varepsilon>0}$ be a family of solutions to ( $\left.E_{\varepsilon}\right)$. For every $\varepsilon_{k} \rightarrow 0$ there are a subsequence $\varepsilon_{k_{j}} \rightarrow 0$ and $u_{0} \in C^{0,1}(\bar{\Omega})$ such that

(1) $u^{\varepsilon_{k_{j}}} \rightarrow u_{0}$ uniformly in $\bar{\Omega}$,

(2) $F\left(X, \nabla u_{0}, D^{2} u_{0}\right)=0$ in $\Omega \cap\left\{u_{0}>0\right\}$ in the viscosity sense. 


\section{Appendix}

In this final section we are going to give the proof of some results, which were temporarily omitted. LEMMA 2.9 (Boundary's estimates propagation Lemma) Suppose that $u \geqslant 0$ is a viscosity solution to

$$
\left\{\begin{aligned}
F\left(X, \nabla u, D^{2} u\right)=0 & \text { in } \quad B_{1}^{+}, \\
u \geqslant \sigma>0 & \text { on } \quad B_{1}^{\prime} .
\end{aligned}\right.
$$

Then there exists a universal constant $C=C(n, \lambda, \Lambda, b)>0$ such that

$$
u(X) \geqslant C \sigma, \quad X \in B_{3 / 4}^{+}
$$

Proof. First of all consider the following Dirichlet problem

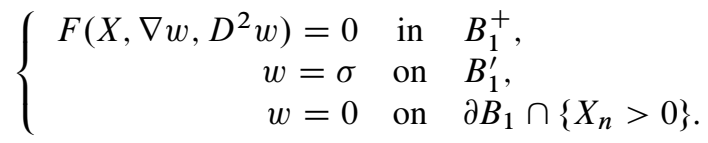

From $C^{1, \alpha}$ regularity estimate we have $w \in C^{1, \alpha}\left(\bar{B}_{3 / 4}^{+}\right)$, and, by the Comparison Principle

$$
0 \leqslant w \leqslant \sigma \quad \text { in } B_{1}^{+}
$$

From now on, it is appropriate we define the following reflection $U: B_{1} \rightarrow \mathbb{R}$,

$$
G(X, \vec{p}, M):=\left\{\begin{array}{rll}
F(X, \vec{p}, M) & \text { if } \quad X_{n} \geqslant 0 \\
-F(\widetilde{X}, \overrightarrow{\widetilde{p}}, \widetilde{M}) & \text { if } \quad X_{n}<0,
\end{array}\right.
$$

We observe that $U$ is a viscosity solution to

$$
G\left(X, D U, D^{2} U\right)=0 \quad \text { in } \quad B_{1},
$$

where

$$
G(X, \vec{p}, M):=\left\{\begin{array}{rll}
F(X, \vec{p}, M) & \text { if } & X_{n}>0, \\
-F(\widetilde{X}, \vec{p}, \widetilde{M}) & \text { if } & X_{n}<0
\end{array}\right.
$$

with

$$
\begin{gathered}
\widetilde{X}=\left(X_{1}, \ldots, X_{n-1},-X_{n}\right), \\
\widetilde{p}=\left(-p_{1}, \ldots,-p_{n-1}, p_{n}\right), \\
\widetilde{M}=\left\{\begin{array}{rc}
-M_{i j} & \text { if } \quad 1 \leqslant i, j \leqslant n-1 \\
M_{i j} & \text { otherwise. }
\end{array} \text { or } i=j=n\right.
\end{gathered}
$$

Thus, from (2.18),

$$
\sigma \leqslant U \leqslant 2 \sigma \quad \text { in } \quad B_{1}^{-} .
$$

Hence,

$$
0 \leqslant U \leqslant 2 \sigma \quad \text { in } \quad B_{1} .
$$


Moreover, from Harnack inequality we have that

$$
\sup _{B_{3 / 4}} U \leqslant c_{0} \inf _{B_{3 / 4}} U
$$

and, in particular,

$$
w(X) \geqslant c_{0}^{-1} \sigma \quad \text { in } \quad B_{3 / 4}^{+} .
$$

Therefore, the proof follows through the previous inequality combined with the Comparison Principle.

LEMMA 2.10 (Hopf's type boundary principle) Let $u$ be a viscosity solution to

$$
\left\{\begin{aligned}
F\left(X, \nabla u, D^{2} u\right)=0 & \text { in } \quad B_{r}(Z) \\
u \geqslant 0 & \text { in } \quad B_{r}(Z)
\end{aligned}\right.
$$

with $r \leqslant 1$. Assume that for some $X_{0} \in \partial B_{r}(Z)$,

$$
u\left(X_{0}\right)=0 \quad \text { and } \quad \frac{\partial u}{\partial v}\left(X_{0}\right) \leqslant \theta
$$

where $v$ is the inward normal direction at $X_{0}$. Then there exists a universal constant $C>0$ such that

$$
u(Z) \leqslant C \theta r .
$$

Proof. By using a scaling argument, we may assume $r=1$. Indeed, it is sufficient to consider the scaled function $v: B_{1} \rightarrow \mathbb{R}$

$$
v_{r}(Y)=\frac{u(X+r Y)}{r}
$$

As before, $v_{r}$ is a viscosity solution of

$$
F_{r}\left(Y, \nabla v_{r}, D^{2} v_{r}\right)=0 \quad \text { in } \quad B_{1},
$$

with

$$
F_{r}(Y, \vec{p}, M):=r F\left(X+r Y, \vec{p}, r^{-1} M\right)
$$

Let $A:=B_{1} \backslash B_{1 / 2}$ be an annular region and define $\omega: A \rightarrow \mathbb{R}$ by

$$
\omega(Y):=\mu\left(e^{-\delta|Y|^{2}}-e^{-\delta}\right)
$$

where the positive constants $\mu$ and $\delta$ will be chosen a posteriori. One can computer the gradient and Hessian of $\omega$ in $A$ as follows

$$
\begin{aligned}
\partial_{i} \omega(Y) & =-2 \mu \delta Y_{i} e^{-\delta|Y|^{2}}, \\
\partial_{i j} \omega(Y) & =4 \mu \delta^{2} Y_{i} Y_{j} e^{-\delta|Y|^{2}}-2 \mu \delta e^{-\delta|Y|^{2}} \delta_{i j}, \\
|\nabla \omega(Y)| & =2 \mu \delta e^{-\delta|Y|^{2}}|Y| .
\end{aligned}
$$


In particular, for every $M \in Q_{\lambda, \Lambda}:=\left\{A \in \operatorname{Sym}(n): \lambda\|\xi\|^{2} \leqslant A_{i j} \xi_{i} \xi_{j} \leqslant \Lambda\|\xi\|^{2}, \forall \xi \in \mathbb{R}^{n}\right\}$ we have

$$
\begin{aligned}
\operatorname{Tr}\left(M \cdot D^{2} \omega\right)-b|\nabla \omega| & =\sum_{i, j=1}^{n} m_{i j} \partial_{i j} \omega-b \cdot \sqrt{\sum_{i=1}^{n}\left(\partial_{i} \omega\right)^{2}} \\
& =4 \mu \delta^{2} e^{-\delta|Y|^{2}} \operatorname{Tr}(M \cdot Y \otimes Y)-2 \delta \mu \operatorname{Tr}(M) e^{-\delta|Y|^{2}}-2 \mu \delta b|Y| e^{-\delta|Y|^{2}} \\
& \geqslant 4 \mu \delta^{2} \lambda|Y|^{2} e^{-\mu|Y|^{2}}-2 \delta \mu n \Lambda e^{-\delta|Y|^{2}}-2 \mu \delta b|Y| e^{-\delta|Y|^{2}} \\
& =2 \mu \delta\left(2 \delta \lambda|Y|^{2}-b|Y|-n \Lambda\right) e^{-\delta|Y|^{2}} \\
& \geqslant 2 \mu \delta\left(\frac{\delta \lambda}{2}-b-n \Lambda\right) e^{-\delta|Y|^{2}} \quad \text { in } \quad A,
\end{aligned}
$$

where $\xi \otimes \xi=\left(\xi_{i} \xi_{j}\right)_{i, j}$. Choose and fix $\delta \geqslant \frac{2}{\lambda}(b+n \Lambda)$. Then, it follows readily that

$$
\mathbb{P}_{\lambda, \Lambda}^{-}\left(D^{2} \omega\right)-b|\nabla \omega| \geqslant 0 \text { in } A .
$$

Therefore, since $r \leqslant 1$, if $\delta \in\left[\frac{2}{\lambda}(\tilde{b}+n \Lambda),+\infty\right)$, with $\tilde{b}=r b$, we have

$$
F_{r}\left(X, \nabla \omega(X), D^{2} \omega(X)\right) \geqslant 0 \quad \text { in } \quad A .
$$

Now by Harnack inequality

$$
v_{r}(0) \leqslant \sup _{B_{1 / 2}} v_{r} \leqslant c_{0} \inf _{B_{1 / 2}} v_{r}
$$

By choosing $\mu=\frac{v_{r}(0)}{c_{0}\left(e^{-\delta / 4}-e^{-\delta}\right)}$ we have $\omega \leqslant v_{r}$ on $\partial A$ and via Comparison Principle gives that $\omega \leqslant v_{r}$ in $A$. Thus, if we label $Y_{0}:=\frac{X_{0}-Z}{r}$ then

$$
\mu \delta e^{-\delta} \leqslant \frac{\partial \omega}{\partial v}\left(Y_{0}\right) \leqslant \frac{\partial v_{r}}{\partial v}\left(Y_{0}\right) \leqslant \theta .
$$

Therefore,

$$
v(0) \leqslant \theta \delta^{-1} c_{0}\left(e^{\frac{3 \delta}{4}}-1\right),
$$

and by returning to the original sentence we can conclude that

$$
u(Z) \leqslant c \theta r .
$$

Acknowledgements. The authors would like to thank Eduardo V. Teixeira for insightful comments and suggestions that benefited a lot the final outcome of this article. We would like to thank anonymous referees by the careful reading and suggestions throughout the paper. This article is part of the second author's PhD thesis which would like to thank Department of Mathematics at Universidade Federal do Ceará-FC -Brazil for fostering a pleasant and productive scientific atmosphere during the period of his $\mathrm{PhD}$ program. This work was partially supported by CAPESBrazil, CNPq-Brazil and FUNCAP-Ceará. 


\section{REFERENCES}

1. Alt, H. W. \& CAffarelli, L. A., Existence and regularity for a minimum problem with free boundary. J. Reine Angew. Math. 325 (1981), 105-144. Zbl0449. 35105 MR0618549

2. Berestycki, H., CAffarelli, L. \& Nirenberg, L., Uniform estimates for regularization of free boundary problems. Analysis and Partial Differential Equations, Lectures Notes in Pure and Appl. Math., Vol. 122, Dekker, New York, 1990, 567-619. Zb10702.35252 MR1044809

3. Caffarelli, L. A. \& CABré, X., Fully nonlinear elliptic equations. American Mathematical Society Colloquium Publications, Vol. 43. American Mathematical Society, Providence, RI, 1995. Zbl0834. 35002 MR1351007

4. Gurevich, A., Boundary regularity for free boundary problems. Comm. Pure Appl. Math. 52 (1999), 363-403. Zb10928. 35213 MR1656068

5. KARAKHANYAN, A., Up-to boundary regularity for a singular perturbation problem of p-Laplacian type. J. Differential Equations 226 (2006), 558-571. Zb11159. 35350 MR2237691

6. Ricarte, G. \& Teixeira, E., Fully nonlinear singular perturbed equations and asymptotic free boundaries. J. Funct. Anal. 2616 (2011), 1624-1673. Zbl1261. 35059 MR2813483

7. TEIXEIRA, E., Optimal regularity of viscosity solutions of fully nonlinear singular equations and their limiting free boundary problems. XIV School on Differential Geometry (Portuguese). Mat. Contemp. 30 (2006), 217-237. Zbl1159.49037 MR2373512

8. WINTER, N., $W^{2, p}$ and $W^{1, p}$-estimates at the boundary for solutions of fully nonlinear, uniformly elliptic equations. Z. Anal. Adwend. (J. Anal. Appl.) 28 (2009), 129-164. Zbl1206.35116 MR2486925 